\title{
GERDA, the GERmanium Detector Array for the search of neutrinoless beta decay of Ge-76
}

\author{
Karl Tasso Knöpfle* representing the GERDA collaboration \\ MPI Kernphysik, Heidelberg, Germany \\ E-mail: Karl-Tasso.Knoepfle@mpi-hd.mpg.de
}

\begin{abstract}
GERDA will be a new underground experiment at the Laboratori Nazionali del Gran Sasso (LNGS) with the aim to study neutrinoless double beta $(0 v \beta \beta)$ decay of ${ }^{76} \mathrm{Ge}$ at background levels $\left(<10^{-3} \mathrm{cts} /(\mathrm{keV} \cdot \mathrm{kg} \cdot \mathrm{y})\right.$ at $\left.\mathrm{Q}_{\beta \beta}=2039 \mathrm{keV}\right)$ which are more than two orders of magnitude lower than in existing $0 v \beta \beta$ experiments. Bare Ge-diodes, enriched to $86 \%$ in ${ }^{76} \mathrm{Ge}$, are operated in a $2 \mathrm{~m}$ thick shield of liquid nitrogen or argon complemented by a water shield of $3 \mathrm{~m}$ thickness to reduce the external backgrounds. Intrinsic backgrounds of the diodes will be suppressed by at least a factor of ten by exploiting pulse shape analysis, anti-coincidences between detectors within the array, and, in a later stage, segmented diodes.

In GERDA's first phase, the detector array will consist of the existing and refurbished isotopically enriched ${ }^{76} \mathrm{Ge}$ diodes of the previous HDM and IGEX experiments with a total weight of about $15 \mathrm{~kg}$. After 1 year of measurement, the sensitivity of this setup should allow to scrutinize the recently claimed observation of $0 v \beta \beta$ decay in ${ }^{76} \mathrm{Ge}$ with a lifetime of about $1.2 \cdot 10^{25} \mathrm{y}$ with high statistical significance. Phase II of the GERDA experiment will use an additional set of new enriched and segmented ${ }^{76} \mathrm{Ge}$ detectors. After $100 \mathrm{~kg}$.years and with zero background, the sensitivity will be $\mathrm{T}_{1 / 2}^{0 \mathrm{v}}>2 \cdot 10^{26} \mathrm{y}$ at $90 \%$ confidence level ; this corresponds to an effective Majorana mass range from 0.09 to $0.29 \mathrm{eV}$.
\end{abstract}

The paper will give an overview of the setup, ongoing R\&D and the status of the GERDA experiment which has been approved recently by LNGS.

International Europhysics Conference on High Energy Physics

July 21st - 27th 2005

Lisboa, Portugal

* Speaker. 
Neutrino experiments have provided recently convincing evidence that neutrinos do have a non-zero mass and that they do oscillate. Further progress can come from the study of neutrinoless double beta $(0 v \beta \beta)$ decay which can yield information on the absolute neutrino mass scale, and which is the only means to answer the fundamental question if the neutrino is - unlike any other fermion - its own antiparticle. The best limits for $0 v \beta \beta$ decay were for a long time due to the Heidelberg-Moscow (HDM) and IGEX enriched ${ }^{76} \mathrm{Ge}$ experiments $[1,2]$ yielding lower half-life limits of about $\mathrm{T}_{1 / 2}^{0 v}>1.6 \cdot 10^{25}$ y and corresponding effective Majorana masses of $m_{\beta \beta}<0.33$ $1.35 \mathrm{eV}$ where the range of $m_{\beta \beta}$ values reflects the estimated uncertainties in the nuclear matrix elements needed to convert $\mathrm{T}_{1 / 2}^{0 v}$ into $m_{\beta \beta}$. Based on an enlarged data sample of $72 \mathrm{~kg} \cdot$ years, a fraction of the HDM collaboration has claimed recently the observation of $0 v \beta \beta$ decay in ${ }^{76} \mathrm{Ge}$ with an half-life of $\mathrm{T}_{1 / 2}^{0 v}=1.2_{-0.5}^{+3.0} \cdot 10^{25} \mathrm{y}$, implying a $m_{\beta \beta}$ value between 0.1 and $0.9 \mathrm{eV}$ with the central value of $0.44 \mathrm{eV}$ [3]. In view of the controversial aspects of this result (see e.g [4]) scrutiny by other more sensitive experiments is needed. The ongoing experiments CUORICINO and NEMO3 could confirm the $0 v \beta \beta$ signal with ${ }^{130} \mathrm{Te}$ and ${ }^{100} \mathrm{Mo}$ but cannot refute the claim in case of a null result due to the uncertainties of the nuclear matrix elements. In fact, CUORICINO has reported most recently a null-result with the upper bound of $m_{\beta \beta}<0.2-1.1 \mathrm{eV}[5]$ ! - The GERDA experiment $[6,7]$ aims at probing $0 v \beta \beta$ decay of ${ }^{76} \mathrm{Ge}$ with a sensitivity of $\mathrm{T}_{1 / 2}^{0 v}>2 \cdot 10^{26} \mathrm{y}$ at $90 \%$ confidence level corresponding to a $m_{\beta \beta}$ range from 0.09 to $0.29 \mathrm{eV}$. Using in its first phase the refurbished ${ }^{76} \mathrm{Ge}$ detectors (a total of 15 to $20 \mathrm{~kg}$ ) of the previous HDM and IGEX experiments, GERDA will be able to scrutinize the recent claim for the $0 v \beta \beta$ decay observation with high statistical significance after one year of running. GERDA will reach its ultimate sensitivity in Phase II where the total ${ }^{76} \mathrm{Ge}$ mass will be increased to more than $30 \mathrm{~kg}$ by adding new enriched ${ }^{76} \mathrm{Ge}$ detectors of improved design.
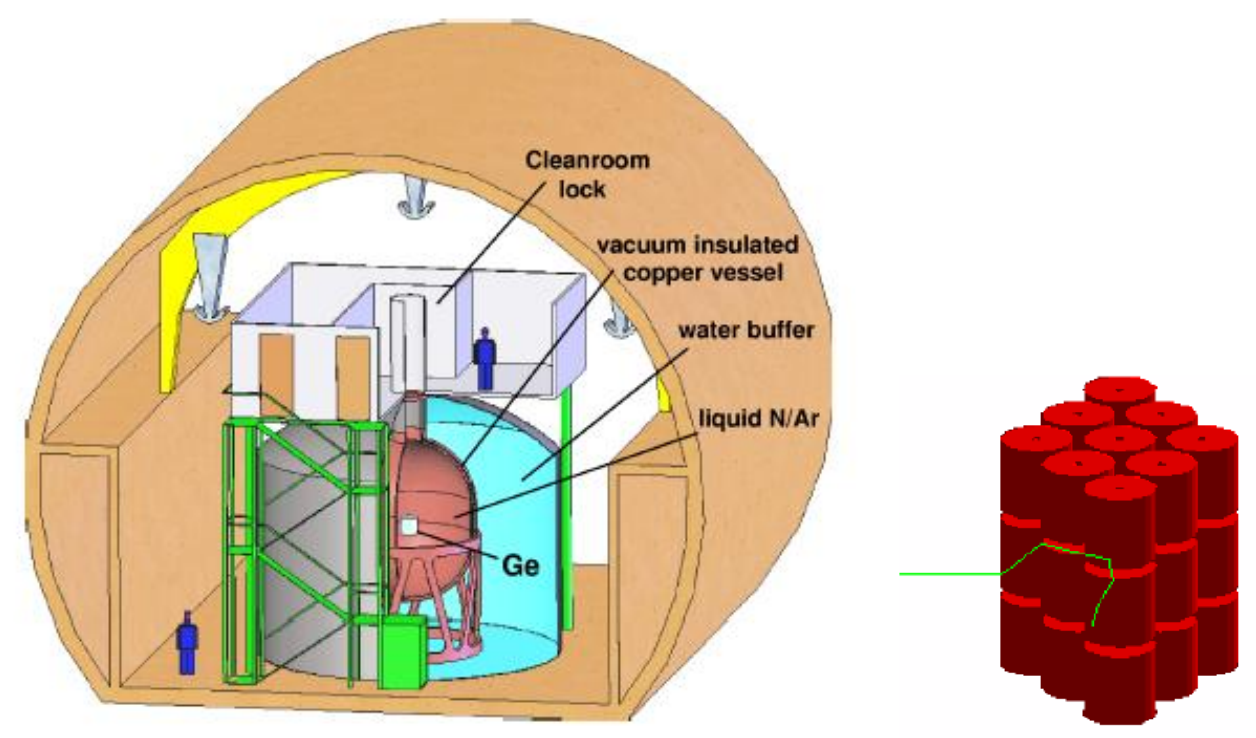

Figure 1: Left: Artist's view of the GERDA experiment in Hall A of LNGS. Right: The array of 27 Ge diodes ( $2 \mathrm{~kg}$ each, $\mathrm{H} \times \varnothing=78 \times 78 \mathrm{~mm}$ ) used in the Monte Carlo simulation. The green line exemplifies the interaction of a $2.6 \mathrm{MeV} \gamma$ ray in the array. 
The layout of the GERDA experiment (Fig. 1) is similar to the GEM proposal [8]. As suggested earlier [9], bare Ge diodes are operated in an ultra-pure liquefied gas, nitrogen (LN) or argon (LAr), which acts both as cooling and shielding medium. The cryogenic fluid is contained in a vacuumisolated cryostat; its diameter of about $4 \mathrm{~m}$ has been chosen such that the $\mathrm{LN}$ shields the activity $\left(<20 \mu \mathrm{Bq} / \mathrm{kg}^{228} \mathrm{Th}\right)$ of the cryostat walls which are made from special OFE copper. An outer about $3 \mathrm{~m}$ thick layer of highly purified water $\left(\sim 1 \mu \mathrm{Bq} / \mathrm{kg}{ }^{228} \mathrm{Th}\right)$ complements the shielding against the rock and concrete $\left(\sim 3 \mathrm{~Bq} / \mathrm{kg}{ }^{228} \mathrm{Th}\right)$. This water buffer serves also as a neutron shield and instrumented with photomultipliers - as Cherenkov medium for vetoing cosmic muons. The Ge detector array has a hexagonal structure (see r.h.s. of Fig. 1) and is made up of individual detector strings. A cleanroom and lock on top of the vessel system allow to insert and remove individual detector strings without contaminating the cryogenic volume. Similarly, calibration sources can be brought close to the array. A detector string is obtained by connecting up to five independent detector modules. Prototypes for such detector modules are shown in Fig. 2 for Phase I and II.
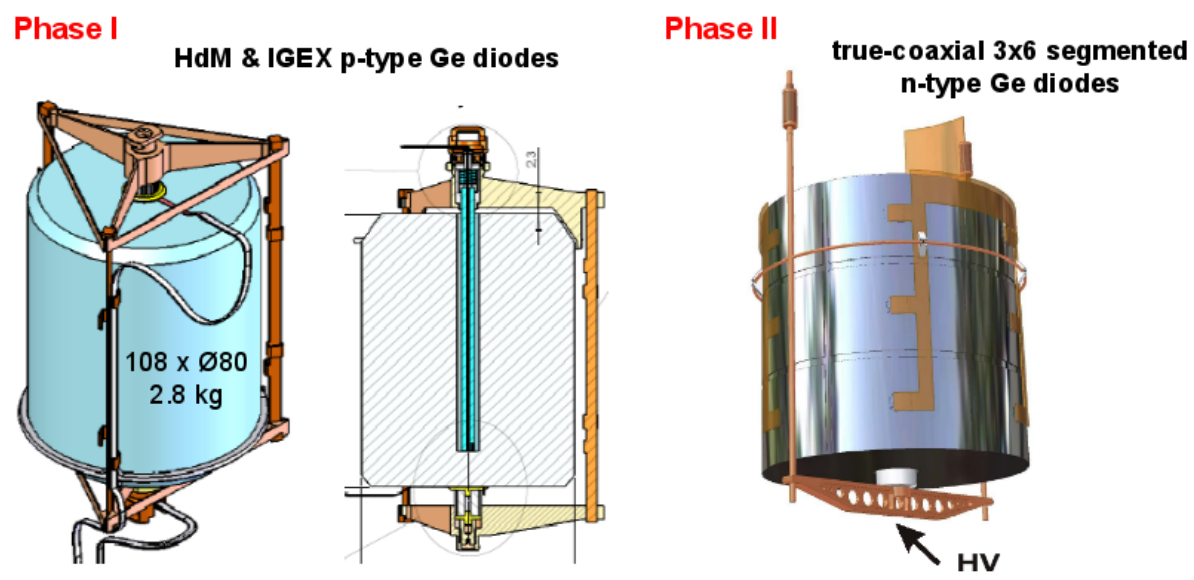

Figure 2: Detector support and contacts for the existing (Phase I) and for the new segmented (Phase II) enriched ${ }^{76} \mathrm{Ge}$ diodes. For the p-type diodes of Phase I, the signal contact at the bottom of the central hole is realized by a pin and spring made both from silicon. The true-coaxial n-type diodes of Phase II have a segmented outer electrode whose contacts are connected to a Teflon cable by ultrasonic wire bonding.

The intrinsic backgrounds of the Ge diodes need also to be reduced in order to yield the desired level of sensitivity. The most relevant contributions are known to come from ${ }^{60} \mathrm{Co}$ and ${ }^{68} \mathrm{Ge}$ since their lifetimes are in the order of years and their decay chains exhibit Q-values above the $\mathrm{Q}_{\beta \beta}$ value. Among others, these isotopes are produced by cosmogenic spallation in the germanium, and hence, an obvious way for their reduction is to reduce the exposure of the enriched Ge material to (hadronic) cosmic rays as much as possible. This recipe is being followed in the procurement of new enriched detectors. Other methods for intrinsic background suppression will exploit that $\beta \beta$ decay has a point-like energy deposition (i.e. a 'single-site' event) while the ${ }^{60} \mathrm{Co}$ and ${ }^{68} \mathrm{Ge}$ decays lead to extended events due to Compton interactions. Such 'multi-site' events will be suppressed by anti-coincidence of detectors within the array or - due to higher granularity even more efficiently - in segmented detectors. Another complementary method is to identify multi-site events from the time structure of the signal. With LAr as cryogenic fluid, background may be suppressed very 
efficiently by simultaneously measuring the scintillation light of the LAr.

Current R\&D activities are of broad range: (i) Mechanical engineering focuses on the preparation for the clean manufacture of the copper cryostat as well as on the development of low-mass suspension and contact systems for the Ge diodes. For Phase II detector modules (see Fig. 2), the total amount of materials for support and contacts - exclusively copper and Teflon - will be less than 30 g. (ii) Electronic engineering has established a working frontend with a cold FET close to the Ge diode and a warm / cold preamplifier outside / inside the cryostat which is acceptable for Phase I. For Phase II, shorter rise time and more compact physical dimensions are required, and two ASIC CMOS chips are under development which are designed to provide the required bandwidth (20 - $30 \mathrm{MHz}$ ) and equivalent noise charge ( $<100 \mathrm{e}^{-}$at $30 \mathrm{pF}$ load) at $\mathrm{LN}$ temperature ( $\left.77 \mathrm{~K}\right)$. (iii) Extensive Monte Carlo simulations are used to study the effects of external $\gamma$, neutron, and muon backgrounds, to determine the allowed radioactivity for the materials used to mount and contact the Ge diodes, as well as to find optimum detector geometry and algorithms for the discrimination of single- and multi-site events. (iv) The radiopurity of materials to be used for GERDA is assessed by $\gamma$ spectrometry, ICP-MS, neutron activation, and $\alpha$ counting. Further work addresses the purity control of LN and LAr in terms of ${ }^{222} \mathrm{Rn}$, surface impurity assessment, and purification procedures. (v) The procurement of new ${ }^{76} \mathrm{Ge}$ diodes has started with the production of 37.5 $\mathrm{kg}$ of enriched $(>86 \%){ }^{76} \mathrm{Ge}$ material, and major effort is devoted to optimize also the following steps including the shielded transport of the material, its chemical purification, zone refinement and the crystal production. The new diodes will be true-coaxial n-type crystals with segmented outer electrode. Test facilities and methods for the characterization of these are under development.

The GERDA collaboration was constituted in February 2004 and comprises now about 70 physicists coming from 13 institutions of five countries. The Letter of Intent [6] was submitted to LNGS in March followed by the Proposal [7] in September 2004. LNGS has approved GERDA in February 2005 and allocated space for it in Hall A in front of the LVD detector. The safety concept of GERDA has been reviewed and accepted after agreement on the implementation of an additional wall between cryostat and water vessel for further mitigation of possible risks from cryostat and water vessel. The start of construction in Hall A is expected for the summer of 2006, the commissioning and the start of the first physics run in 2007.

\section{References}

[1] H.V. Klapdor-Kleingrothaus et al., Eur. Phys. J. A 12 (2001) 147-154 [hep-ph/0103062].

[2] C.E. Alseth et al., Phys. Rev. D 65 (2002) 092007 [hep-ex/0202026].

[3] H.V. Klapdor-Kleingrothaus, I.V. Krivosheina, A. Dietz, and O. Chkvorets, Phys. Lett. B 586 (2004) 198-212 [hep-ph/0404088].

[4] A. Strumia and F. Vissani, Nucl. Phys. B 726 (2005) 294-316 [hep-ph/0503246].

[5] C. Arnaboldi et al., Phys. Rev. Lett. 95 (2005) 142501 [hep-ex/0501034].

[6] The Gerda Collaboration, Letter of Intent (2004) [hep-ex/04040390].

[7] The GerDa Collaboration, Proposal (2004) http://www.mpi-hd.mpg.de/GERDA/proposal.pdf .

[8] Yu.G. Zdesenko, O.A. Ponkratenko, and V.I. Tretyak, J. Phys. G 27 (2001) 2129 [nucl-ex/0106021].

[9] G. Heusser, Ann. Rev. Nucl. Part. Sci. 45 (1995) 543. 